\title{
Safety and Efficacy of Pediatric General Anesthesia by Laryngeal Mask Airway without Intravenous Access
}

\author{
Benjamin A. Lin ${ }^{1}$, Zakaria S. Messieha ${ }^{2 *}$, William E. Hoffman ${ }^{3}$ \\ ${ }^{1}$ Chief resident, Oral and Maxillofacial Surgery, University of Illinois, Chicago, USA; ${ }^{2}$ Associate Professor, Anesthesiology, Univer- \\ sity of Illinois, Chicago, USA; ${ }^{3}$ Research Associate Professor, Anesthesiology, University of Illinois, Chicago, USA. \\ Email: ${ }^{*}$ messieha@uic.edu
}

Received January $9^{\text {th }}, 2011$; revised May $29^{\text {th }}, 2011$; accepted June $7^{\text {th }}, 2011$.

\begin{abstract}
The safety and efficacy of the laryngeal mask airway (LMA) has been reported by numerous large-scale studies. However, they do not address the issue of whether an intravenous (IV) is required for pediatric general anesthesia (GA) where access is challenging due to anatomical considerations and a lack of cooperation. The aims of this study are to determine whether pediatric GA by LMA without IV access affected placement rates, procedure times and rates of anesthetic complications. Children who met these criteria at the UIC Surgicenter in the two year period prior to August 30, 2005 were selected. A retrospective chart review was conducted to determine patient demographics, ASA class, procedure, placement success, IV placed if any, time to incision, and any anesthetic complications. 241 patients without IV access and 41 patients with IV access were included. No significant differences were found between the groups in the rates of LMA placement or anesthetic complications. Significant differences were found in times to incision overall and for ophthalmology exams under anesthesia and lacrimal duct probings. Pediatric GA by LMA without IV access demonstrated a similarly high placement rate, shorter procedure times and a low rate of complications in comparison with the control group.
\end{abstract}

Keywords: Pediatric Anesthesia, Anesthesia Efficacy, LMA Safety, General Anesthesia without Intravenous Cannulation

\section{Introduction}

The laryngeal mask airway (LMA) is a device used to secure an airway in an unconscious patient and is widely and routinely used in the operating room for general anesthesia (GA). GA in children differs than that for adults as children tend not to tolerate the placement of an intravenous (IV) line pre-operatively, which would allow for the injection of medication. In addition to being uncooperative, children possess veins that are correspondingly smaller than that of an adult making IV placement technically more challenging. Typically, an IV is placed once the child is asleep, depending on the preference of the anesthesiologist. Elimination of this step entirely would shorten operating room time and be more cost effective.

There are no published accounts of GA by LMA used in the specific context of no IV access; however, the safety and efficacy of GA by LMA is well established by the literature. In a retrospective study, Verghese et al. describe the successful use of the LMA with IV access in 11,910 adults and children with an overall placement success rate of $99.81 \%$, a risk of laryngospasm of $0.07 \%$, bronchospasm of $0.025 \%$, and vomiting of $0.017 \%$ [1]. Mason et al. describe in a prospective study, the use of the LMA with either Halothane or Isoflurane in 200 children with IV access for a variety of surgical procedures with a successful placement rate of $97.5 \%$, laryngospasm in $2.5 \%$ and vomiting in $1.5 \%$ [2]. Lopez-Gil et $a l$. in another prospective study consisting of 1400 children with LMA usage with Isoflurane and IV access found successful placement in $98 \%$ of patients, $2.7 \%$ with upper airway stimulation, including laryngospasm/ bronchospasm and vomiting/regurgitation/aspiration in $0.07 \%$ [3]. Conclusions of this study were that the incidence of problems is similar to those of adults but that there were a significantly higher complication rate $(p<$ 
0.001) for ENT procedures and when using the size 1 LMA.

There has been only one report in the literature (Haupert et al. 2004) that addresses the issue of whether an IV is even required for pediatric general anesthesia where access is technically challenging due to anatomical considerations and a lack of patient cooperation. This randomized controlled study had 100 ASA statuses I or II children aged 2-12 who underwent bilateral myringotomies with pressure equalizing tube placement [4]. One group received IV access and the other did not. Both groups had mask induction and maintenance with oxygen, nitrous oxide, and Sevoflurane. All children received Fentanyl intramuscularly and spontaneous ventilation was maintained. Patients with IV access received Lactated Ringer's solution. Their study concluded that intravenous access in otherwise healthy children in myringotomy procedures provided no added benefit [4]. Significant differences included more pain medication required $(p<0.001)$, lower parental satisfaction $(p<$ $0.001)$ and more time spent in both the operating room $(p=0.02)$ and recovery $(p=0.02)$ for the group of children with IV access [4].

The purpose of this study was to determine if general anesthesia by laryngeal mask airway without intravenous access is safe and efficacious, resulting in highly successful placement rates, shorter procedure times and a low rate of anesthetic complications in a pediatric population. Our study would be the first to highlight the benefits of using general anesthesia by LMA without IV access for short pediatric procedures in the head and neck region such as bilateral myringotomies and tube placement (BMT), ophthalmologic exams under anesthesia (EUA), nasolacrimal duct probing and chalazion excision.

\section{Methods}

After receiving institutional review board approval, the surgery schedule at the University of Illinois Medical Center Surgicenter was used to select patients aged 0-17 who underwent GA by LMA between the dates of September 1, 2003 to August 30, 2005. A retrospective chart review was performed and the following documents were gathered for each potential subject: anesthesia records, operative reports, and if applicable, Surgicenter reports, hospital admission notes and recovery room notes. Subjects having complete records were then chosen for inclusion. Subjects lacking the appropriate documentation were excluded from the study. Eligible patients were then assigned into 2 groups, based on whether they had an IV placed after mask induction with Sevoflurane. Group 1 had no IV access, and group 2 had IV access. The following data was then collected and compared between the two groups:

- Age, gender, weight, medical conditions and ASA class

- Procedure done and operating room (OR) time to incision

- LMA type used and whether successfully placed

- Type of anesthetic used, method of ventilation

- Type and location of intravenous placed if any

- Medications given pre-, peri- and post-operatively and route of administration

- Anesthesia complications and management

Statistical analysis was performed using the software program SigmaStat for Windows. Z-tests were utilized for analysis of both LMA placement success rates and complication rates between the groups 1 and 2. T-tests were utilized for analysis of time to incision between the groups. Statistically significant differences were considered achieved at $p<0.05$.

\section{Results}

A total of 282 eligible pediatric patients were included in this study. Group 1 had 241 patients and group 2 had 41 patients. $43.2 \%$ of the patients in group 1 and $46.3 \%$ of the patients in group 2 were female. Figure 1 shows the age distribution of the patients in each group. Figure 2 shows the distribution of patients according to American Society of Anesthesiologists (ASA) classification.

Table 1 shows the placement success rates between the groups and according to LMA size as well as overall. Group 1 had a $99.6 \%$ rate of successful LMA placement while group 2 had a $97.6 \%$ success rate. There was no significant difference between the groups in terms of overall LMA placement success rates by Z-test $(p=$ $0.63)$.

Table 2 lists the mean OR time to incision for each of the procedures and overall performed between the two

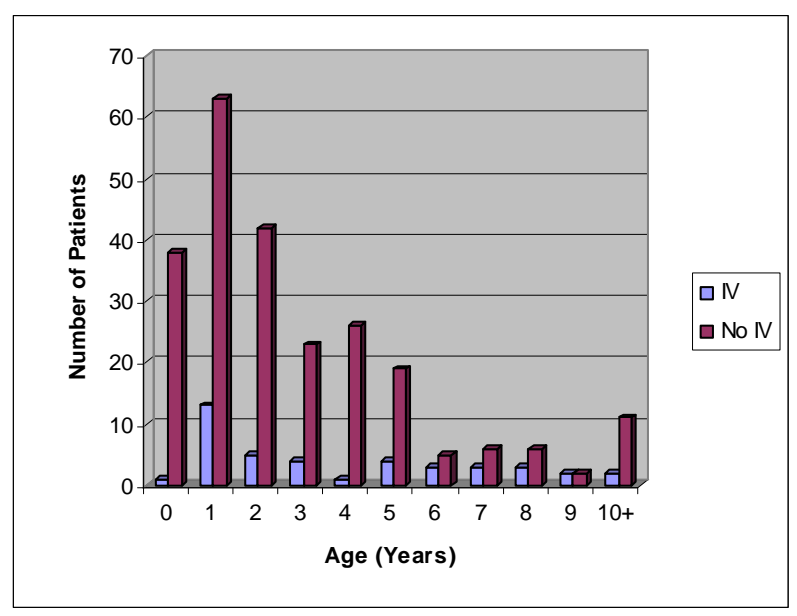

Figure 1. Age distribution. 


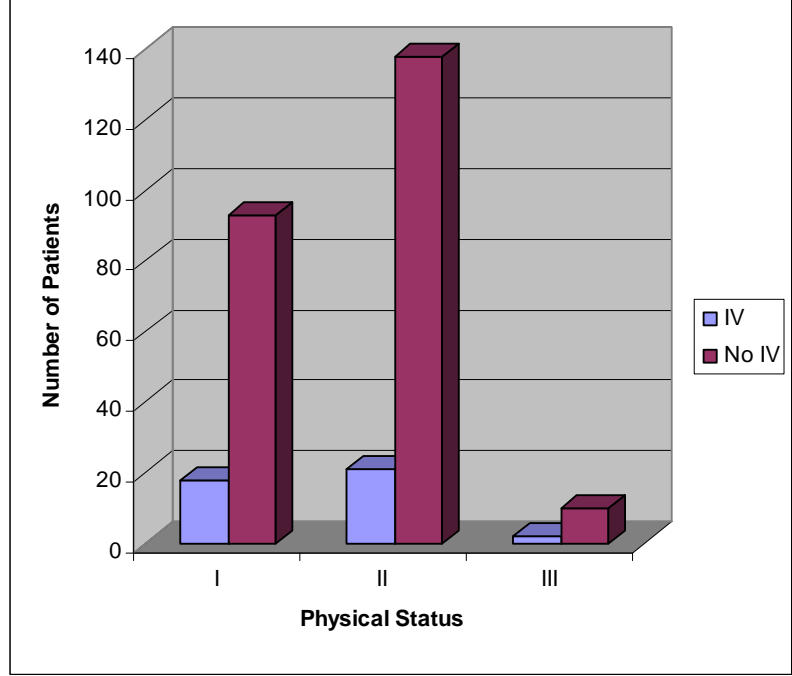

Figure 2. ASA distribution.

groups as well as the mean time saved. There were significant differences between the two groups for the procedures EUA $(p=0.02)$ and lacrimal duct probing $(p<$ $0.001)$ as well as overall $(p<0.001)$. No statistical difference was observed for BMT $(p=0.07)$ and chalazion excision $(p=0.47)$.

Table 3 shows the types and overall rate of anesthetic complications by group. Group 1 had a 1.66\% complication rate while group 2 had a $4.88 \%$ complication rate. There was no significant difference between the groups in terms of overall anesthetic complication rates by Z-test $(p=0.47)$.

\section{Discussion}

Children represent a unique challenge to the anesthesiologist due to their lack of co-operation and difficult anatomy. Placement of an IV is not innocuous and multiple attempts at establishing one can take up a significant amount of OR time as well as introduce additional sites for post operative pain and scarring.

Our institution has been performing EUAs, BMTs, nasolacrimal duct probings and chalazion excisions without IV access for numerous years. Our patient demographics show that the majority of our cases were under the age of 6 and ASA class I or II.

Our retrospective chart review demonstrated that there was no significant difference between the groups in terms of overall LMA placement success rates and that the rates were comparable to those found in the literature. This is understandable since all of the LMAs in our study, regardless of IV status, were placed after mask induction with Sevoflurane. One of the advantages of the LMA is that muscle relaxation is not required for placement [5]. In the rare event that the LMA could not
Table 1. LMA placement success rates.

\begin{tabular}{lllll}
\hline $\begin{array}{l}\text { LMA } \\
\text { Size }\end{array}$ & No IV $(n=241)$ & $\begin{array}{l}\text { Placement } \\
\text { Success }\end{array}$ & IV $(n=41)$ & $\begin{array}{l}\text { Placement } \\
\text { Success }\end{array}$ \\
\hline 1.5 & $43(17.84 \%)$ & $97.7 \%$ & $3(7.31 \%)$ & $100 \%$ \\
2.0 & $137(56.84 \%)$ & $100 \%$ & $26(63.41 \%)$ & $96.2 \%$ \\
2.5 & $43(17.84 \%)$ & $100 \%$ & $9(21.95 \%)$ & $100 \%$ \\
3.0 & $15(6.22 \%)$ & $100 \%$ & $3(7.31 \%)$ & $100 \%$ \\
4.0 & $2(0.83 \%)$ & $100 \%$ & 0 & N/A \\
5.0 & $1(0.41 \%)$ & $100 \%$ & 0 & N/A \\
Total* & 241 & $99.6 \%$ & 41 & $97.6 \%$ \\
\hline
\end{tabular}

*No significant difference $(p=0.63)$.

Table 2. Times to incision.

\begin{tabular}{|c|c|c|c|c|c|c|}
\hline Procedure & $\begin{array}{l}\text { IV } \\
(n=41)\end{array}$ & $\begin{array}{l}\text { Avg } \\
\text { Time } \\
\text { (min) }\end{array}$ & $\begin{array}{l}\text { No IV } \\
(n=241)\end{array}$ & $\begin{array}{l}\text { Avg } \\
\text { Time } \\
\text { (min) }\end{array}$ & $\begin{array}{l}\text { Avg } \\
\text { Time } \\
\text { Saved } \\
\text { (min) } \\
\end{array}$ & $p$-value \\
\hline $\begin{array}{l}\text { Exam under } \\
\text { Anesthesia }\end{array}$ & $\begin{array}{l}12 \\
29.26 \%\end{array}$ & 14.92 & $\begin{array}{l}167 \\
69.29 \%\end{array}$ & 11.13 & 3.79 & 0.02 \\
\hline $\begin{array}{l}\text { Nasolacrimal } \\
\text { duct probing }\end{array}$ & $\begin{array}{l}7 \\
17.07 \%\end{array}$ & 28.42 & $\begin{array}{l}20 \\
8.29 \%\end{array}$ & 12.55 & 15.87 & $<0.001$ \\
\hline $\begin{array}{l}\text { Chalazion } \\
\text { excision }\end{array}$ & $\begin{array}{l}5 \\
12.19 \%\end{array}$ & 27.4 & $\begin{array}{l}5 \\
2.07 \%\end{array}$ & 23 & 4.4 & 0.47 \\
\hline $\begin{array}{l}\mathrm{B} / 1 \text { myringo- } \\
\text { tomy }+ \\
\text { tube placement }\end{array}$ & $\begin{array}{l}17 \\
41.46 \%\end{array}$ & 15.23 & $\begin{array}{l}49 \\
20.33 \%\end{array}$ & 12.8 & 2.43 & 0.07 \\
\hline Total & 41 & 11.84 & 241 & 18.88 & 7.04 & $<0.001$ \\
\hline
\end{tabular}

Table 3. Anesthetic complication rates.

\begin{tabular}{lll}
\hline & No IV $(n=241)$ & IV $(n=41)$ \\
\hline Complications & $\begin{array}{l}\text { Laryngospasm } \times 3 \\
\text { Bronchospasm } \times 1\end{array}$ & Intra-op regurgitation $\times 1$ \\
Overall Rate*: & $1.66 \%$ & $4.88 \%$ \\
\hline
\end{tabular}

*No significant difference $(p=0.47)$.

be successfully placed, then alternative methods such as mask or endotracheal tube placement were used to maintain general anesthesia.

It is standard at our institution to record time to incision for each procedure by the circulating nurse and this marker was felt to better reflect the time taken for the anesthesiologist to place the LMA without having operative circumstances affect the results. Our study showed that overall, as well as for EUAs and nasolacrimal duct probings, there was a significant difference in time to incisions between the IV and no IV groups. Although not significant for BMTs, it is to be noted that this was barely so and perhaps a larger sample size would have bore this out. There appears to be no significant difference in chalazion excision times to incision but this could have been due to more extensive prepping and draping requirements prior to incision.

There were no significant differences between the two groups in terms of anesthetic complications. The complications were all managed successfully without any 
long term sequelae. In the no IV group, the laryngospasms were managed with jaw thrust and positive pressure ventilation or placement of an IV and an endotracheal tube.

Prior to mask induction of the patient, all intubation equipment, an IV kit and setup and medications including a succinylcholine/atropine dart are prepared.

Although IV is the preferred route for medications, other routes such as intramuscular, tracheal and rectal are available to deliver medications. Our patients were routinely given rectal Acetaminophen or im Ketorolac for post operative analgesia. This also does not preclude us from placing an IV if one is required later on. The disadvantages of not having an IV include the inability to administer fluids. However, our procedures were short and involved minimal to no blood loss. In addition, children possess a greater cardiac reserve and the lack of IV fluids did not affect our hemodynamic stability.

From these results, pediatric GA by LMA without IV access is a feasible option for the anesthesiologist in ASA class I or II patients for short head and neck procedures and demonstrates a high placement rate, shorter procedure times and a low rate of complications in comparison with controls. Future studies could encompass prospective studies, randomization, larger sample sizes, and other procedures such as suture removal.

\section{REFERENCES}

[1] C. Verghese and J. Brimacombe, "Survey of Laryngeal Mask Airway Usage in 11,910 Patients: Safety and Efficacy for Conventional and Nonconventional Usage," Anesthesia and Analgesia, Vol. 82, 1996, pp. 129-133. doi:10.1213/00000539-199601000-00023

[2] D. G. Mason and R. M. Bingham, "The Laryngeal Mask Airway in Children," Anaesthesia, Vol. 45, No. 9, 1990, pp. 760-763. doi:10.1111/j.1365-2044.1990.tb14449.x

[3] M. Lopez-Gil and M. Alvarez, "Safety and Efficacy of the Laryngeal Mask Airway: A Prospective Survey of 1400 Children," Anaesthesia, Vol. 51, No. 10, 1996, pp. 969-972. doi:10.1111/j.1365-2044.1996.tb14968.x

[4] M. S. Haupert, C. Pascual, A. Mohan, B. BarteckaSkrzypek and M. Zestos, "Parental Satisfaction with Anesthesia without Intravenous Access for Myringotomy," Archives of Otolaryngology-Head and Neck Surgery, Vol. 130, No. 9, 2004, pp. 1025-1028. doi:10.1001/archotol.130.9.1025

[5] J. Riazi and D. E. Morrison, "The Laryngeal Mask Airway in Pediatric Anesthesia," Anesthesiology Clinics of North America, Vol. 16, No. 4, 1998, pp. 813-825. doi:10.1016/S0889-8537(05)70062-6 